\title{
Erratum: Effects of proton angular momentum alignment on the two-shears-like mechanism in ${ }^{101}$ Pd [Phys. Rev. C 94, 034305 (2016)]
}

\author{
Zhen-Hua Zhang (张振华)
}

(Q) (Received 12 December 2018; published 13 February 2019)

DOI: 10.1103/PhysRevC.99.029901

There is a mistake in Fig. 8 of the original paper. The angular momentum $J_{x}$ for the proton was doubled in this figure. The corrected Fig. 8 is presented here. The results and conclusions of the original article are not affected.

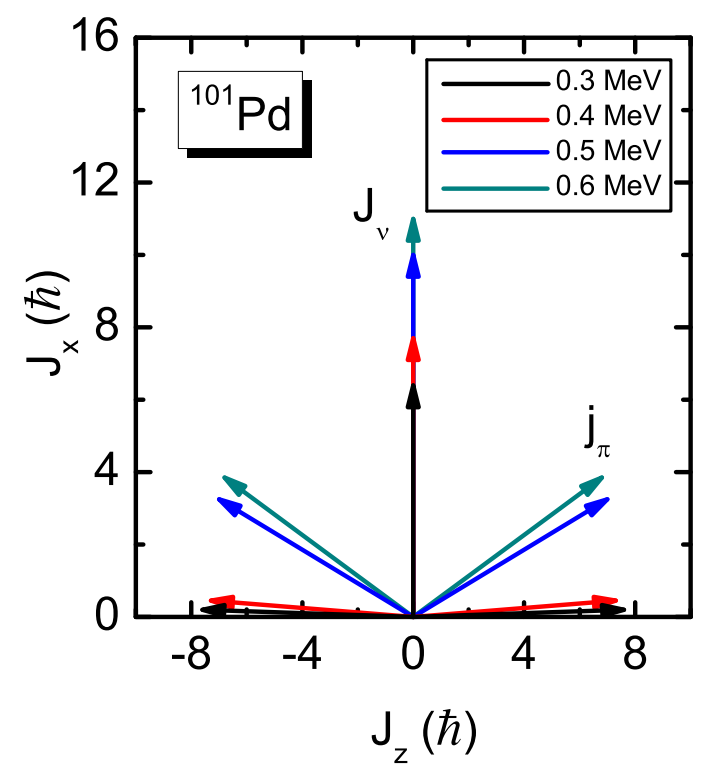

FIG. 8. Angular momentum vectors of neutrons $J_{v}$ and the four $\pi g_{9 / 2}$ proton holes $j_{\pi}$ at rotational frequencies from 0.3 to $0.6 \mathrm{MeV}$ for $v 1 / 2^{-}[550]$ in ${ }^{101} \mathrm{Pd}$. Each proton angular momentum vector contains the contribution of two $g_{9 / 2}$ proton holes. 